\title{
ATUAÇÃO DA FISIOTERAPIA NA URGÊNCIA E EMERGÊNCIA DE UM HOSPITAL REFERÊNCIA EM TRAUMA E QUEIMADOS DE ALTA E MÉDIA COMPLEXIDADE
}

Ingrid da Costa Noronha de ALMEIDA ${ }^{1}$

Gabriela Martins de LIMA ${ }^{2}$

Lorena de Almeida COSTA ${ }^{3}$

Lucas Monteiro CARNEIRO ${ }^{4}$

Maria Isabel Galletti dos SANTOS ${ }^{5}$

Rafaela Cordeiro de MACÊDO ${ }^{6}$

Leonardo Ramos Nicolau da $\operatorname{COSTA}^{7}$

${ }^{1}$ Fisioterapeuta, Residente do Programa de Residência Multiprofissional em Saúde - Saúde da Mulher e da Criança. Universidade do Estado do Pará.ingrid.fisio@yahoo.com.br.

${ }^{2}$ Mestre, Supervisora do Serviço de Reabilitação, Docente do Curso de Fisioterapia. Hospital Metropolitano de Urgência em Emergência, Centro Universitário do Pará. gabimlima@gmail.com.

${ }^{3}$ Fisioterapeuta, Residência Multiprofissional em Saúde em Urgência e Emergência no Trauma. Universidade do Estado do Pará. lorenadeac.2013@gmail.com.

${ }^{4}$ Fisioterapeuta, Especializando em Terapia Intensiva. Universidade de Campinas. lucasmonteiroc02@gmail.com

${ }^{5}$ Fisioterapeuta Residência Multiprofissional em Saúde em Urgência e Emergência no Trauma. Universidade do Estado do Pará.misabelgalletti@ hotmail.com.

${ }^{6}$ Mestre, Fisioterapeuta, Preceptora da Residência em Urgência e Emergência no Trauma, Docente do Curso de Fisioterapia. Hospital Metropolitano de Urgência e Emergência, Centro Universitário do Pará. rafaela_cmacedo@hotmail.com.

${ }^{7}$ Doutorando, Coordenador do Departamento de Ensino e Pesquisa Hospital Metropolitano de Urgência e Emergência, Docente do Curso de Fisioterapia. Universidade Federal do Pará, Universidade do Estado do Pará, Centro Universitário do Estado do Pará, Hospital Metropolitano de Urgência e Emergência, leorcosta@gmail.com.

\section{Recebido em: 23/10/2016 - Aprovado em: 05/05/2017 - Disponibilizado em: 01/07/2017}

\section{RESUMO:}

A inserção do fisioterapeuta nas unidades de urgência e emergência junto à equipe multidisciplinar pode favorecer o atendimento e tratamento dos pacientes que chegam até este tipo de serviço. Objetivou-se identificar a atuação da Fisioterapia no pronto atendimento do Hospital Metropolitano de Urgência e Emergência (HMUE) durante o período de 90 dias, verificando os procedimentos fisioterapêuticos realizados neste setor, bem como o perfil clínico e epidemiológico dos pacientes atendidos por fisioterapeutas neste serviço. Estudo observacional, retrospectivo com estatística descritiva no qual foram analisados prontuários dos pacientes atendidos por fisioterapeutas no pronto atendimento do HMUE. Amostra composta de 175 prontuários de pacientes, a maioria do gênero masculino 82,29\% (144), com faixa etária de 19 a 29 anos (28,57\%), procedentes de Belém-PA 65,14\% (70), submetidos a tratamento clínico conservador $65,14 \%$ (114). O traumatismo cranioencefálico foi o tipo de trauma mais prevalente $65,71 \%$ (115). A região corporal mais acometida pelo trauma foi a cabeça 74,86\% (131) e o mecanismo de lesão mais frequente foi o acidente de moto $32,57 \%$ (57). A classificação de risco vermelho foi a mais observada 62,3\% (109). Os procedimentos fisioterapêuticos mais realizados foram controle de ventilação mecânica $88 \%$ (154) e aspiração endotraqueal $60,57 \%$ (106). O destino da maioria dos pacientes foi a internação na UTI 68,5\% (120). Desta forma, a atuação da Fisioterapia no setor de pronto atendimento visa amenizar os sinais e sintomas clínicos, incluindo os respiratórios a fim de otimizar o tratamento clínico por meio de condutas como controle de ventilação mecânica, aspiração traqueal, montagem de ventilação mecânica, transporte intra-hospitalar, técnicas de fisioterapia respiratória.

Palavras-chave: Fisioterapia. Emergências. Hospitais. Traumatismo. Respiração artificial. 


\section{ABSTRACT:}

The insertion of the physiotherapist in urgency and emergency units in a multidisciplinary team can support the care and treatment of patients who come to this type of service. The objective wasto identify the clinical and epidemiological profile of patients seen by physiotherapists in the emergency service of a referral hospital in trauma and burn high and medium complexity during the 90 days period and check the work of physiotherapists in this service. Observational, retrospective study with descriptive statistics which analyzed medical records of patients seen by physiotherapists in the Emergency Room of the Emergency Hospital Metropolitano and Emergency. The sample was composed of 175 patients records, most males $82.29 \%$ (144), aged 19-29 years (28.57\%), coming from Belém-PA 65.14\% (70), submitted to conservative medical treatment $65.14 \%$ (114). Traumatic brain injury was the most prevalent type of trauma $65.71 \%$ (115). The body region most affected by the trauma was the head $74.86 \%$ (131) and the most common mechanism of injury was the motorcycle accident $32.57 \%$ (57). The red risk rating was the most observed 62.3\% (109). The respiratory therapy procedures were performed more mechanical ventilation control 88\% (154) and endotracheal suctioning $60.57 \%$ (106). The fate of most of the patients were in the ICU 68.5\% (120). Thus the operation of physical therapy in the emergency care sector aims to ameliorate the clinical signs and symptoms, including breathing in order to optimize the clinical treatment through behaviors such as mechanical ventilation control, tracheal suctioning , mechanical ventilation assembly, intra- hospital transport, respiratory therapy techniques. It is necessary to carry out studies to assess the impacts of these activities.

Keywords: Physical Therapy Modalities. Emergencies.HospitalsInjuries.Respiration Artificial.

\section{Introdução}

O serviço de urgência e emergência é a porta de entrada principal de um hospital para o paciente que apresenta alterações importantes do ponto de vista biológico e físico, necessitando de um atendimento imediato, pois apresenta risco eminente de morte (PICCOLI et al, 2013; CAMERRO et al, 2015).

O termo emergência é utilizado quando o paciente apresenta sinais e sintomas que podem indicar risco à vida e requer um atendimento imediato. Já a urgência, é compreendida como a situação onde o paciente necessita de um atendimento rápido, em poucas horas, pois há risco de complicações graves (OGAWA et al, 2009).

Nos últimos anos, houve crescimento da demanda por atendimentos de urgência e emergência. A superlotação neste setor é intensificada por problemas organizacionais como o atendimento por ordem de chegada, sem estabelecimento de critérios clínicos. Ciente dos problemas existentes na atenção às urgências, o Ministério da Saúde preconiza o acolhimento com avaliação e classificação de risco, como dispositivo de mudança no trabalho da atenção à saúde (BRASIL, 2009).

A classificação de risco consiste em um processo dinâmico de identificação depacientes que necessitam de tratamento imediato, de acordo com o potencial derisco, osagravos à saúde ou o grau de sofrimento, devendo o atendimento ser priorizado de acordocom a gravidade clínica do paciente (BRASIL, 2009; ALBINO; GROSSEMAN; RIGGENBACH, 2007).

A assistência para o paciente da urgência e emergência conta atualmente com diversas áreas interligadas para oferecer um melhor atendimento (SIMÔES; URBANETTO; FIGUEIREDO, 2013). Tradicionalmente, o setor de emergência consistia em um tratamento prestado por 
médicos e enfermeiros, especialistas em trauma agudo ou doenças clínicas agudas (PICCOLI et al, 2013).

Atualmente o termo "multidisciplinar" tem sido muito empregado nesses setores, pois agrega todas as especialidades $\mathrm{e}$ variedades de profissionais, que trabalham unidos para um atendimento mais eficaz (SIMÔES; URBANETTO; FIGUEIREDO, 2013). É notória a importância da integração e articulação das diferentes áreas profissionais, pautada na cooperação e troca de saberes em prol de uma assistência adequada aos usuários nas situações de urgência e emergência.

A inserção do fisioterapeuta nas unidades de urgência e emergência é recente, e ainda restrita na grande maioria dos hospitais de alta complexidade. Sua necessidade se dá ao fato do grande número de pacientes possuírem diagnóstico com alteração cardiopulmonar, necessitando de oxigenoterapia e ventilação mecânica (PICCOLI et al, 2013).

A presença do fisioterapeuta no setor de pronto atendimento é indispensável, pois sua atuação junto à equipe assistencial de urgência e emergência pode favorecer $o$ atendimento e tratamento dos pacientes que chegam até este tipo de serviço, contribuindo para a melhora do quadro clínico e diminuição do tempo de permanência destes na emergência (FUKUJIMA,2009).

O conhecimento das competências fisioterapêuticas, pelos pacientes e pelos próprios profissionais da saúde, relacionando a atuação e a reabilitação precoce das patologias, influenciam a gestão de recursos e a alocação dos profissionais. Entretanto, ainda existe uma falta de consciência a respeito da atuação do profissional fisioterapeuta no setor de urgência e emergência (PICCOLI et al ,2013).

Diante disto, este estudo objetivou identificar a atuação da fisioterapia no serviço de pronto atendimento de um hospital referência em trauma e queimados de alta e média complexidade durante o período de 90 dias, verificando os procedimentos fisioterapêuticos realizados neste setor, bem como o perfil clínico e epidemiológico dos pacientes atendidos por fisioterapeutas neste serviço.

\section{Métodos}

Todos os procedimentos dapesquisa foram realizados de acordo com as Normas de Pesquisa Envolvendo Seres Humanos (Res. CNS 466/12) do Conselho Nacional de Saúde, sendo submetido e aprovado pelo Comitê de Ética do Centro Universitário do Pará sob registro CAAE: 4897815.3.0000.5169.

Trata-se de um estudo observacional, retrospectivo com estatística descritiva no qual foram analisados 179 prontuários de pacientes atendidos por fisioterapeutas no pronto atendimento do Hospital Metropolitano de urgência e emergência (HMUE), localizado em Ananindeua-PA, no período de agosto a outubro de 2015. 
A pesquisa foi realizada no setor de arquivo médico e estatística (SAME) do HMUE, no mês de dezembro de 2015. Os dados foram coletados por meio de uma ficha de coleta de dados, elaborada pelos autores do estudo.

As variáveis da pesquisa são: idade, sexo, procedência, tipo de trauma, mecanismo de trauma e região corporal acometida. Bem como, os recursos utilizados no atendimento e o tipo de alta após o atendimento dos fisioterapeutas do HMUE.

Foram excluídos da pesquisa os prontuários de pacientes que não foram atendidos por fisioterapeutas no Pronto Atendimento do HMUE, que não estavam de acordo com o período da pesquisa, assim como os prontuários incompletos.

Após a coleta, os dados foram armazenados em planilha eletrônica excel para o processamento. Foi realizada a análise descritiva dos dados e aplicado o teste Quiquadrado ao nível de significância de 0.05 para verificar a diferença entre as frequências absolutas.

Foram utilizados recursos de computação, por meio do processamento no sistema Microsoft Excel e StatisticPackageforSocial Sciences (SPSS), versão 22.0, todos em ambiente Windows 7.

\section{Resultados}

Foram analisados 179 prontuários, dos quais 04foram excluídos por estarem incompletos. A amostra final foi composta de 175 prontuários.

A tabela 1 mostra a distribuição dos participantes da pesquisa por gênero, faixa etária, procedência e tipo de tratamento clínico recebido. Observa-se que a maioria, $82,29 \%$ (144) dos participantes é do gênero masculino, com faixa etária de 19 a 29 anos (28,57\%), 65,14\% (70) procedentes de Belém e $65,14 \%$ (114) receberam tratamento clínico conservador.

Tabela 1-Frequência e percentual dos participantes da pesquisa por gênero, faixa etária, procedência e tratamento clínico. Belém/PA.

\begin{tabular}{|c|c|c|c|}
\hline Gênero & Frequência & Percentual (\%) & P-valor \\
\hline Feminino & 31 & 17,71 & \multirow{3}{*}{$0,000 *$} \\
\hline Masculino & 144 & 82,29 & \\
\hline Faixa Etária & 175 & 100,00 & \\
\hline 0 a 12 & 8 & 4,57 & \multirow{9}{*}{$0,000^{*}$} \\
\hline 12 a 18 & 13 & 7,43 & \\
\hline 19 a 29 & 50 & 28,57 & \\
\hline 30 a 39 & 38 & 21,71 & \\
\hline 40 a 49 & 25 & 14,29 & \\
\hline 50 a 59 & 15 & 8,57 & \\
\hline Maior que 60 & 25 & 14,29 & \\
\hline Não Informado & 1 & 0,57 & \\
\hline Procedência & 175 & 100,00 & \\
\hline Ananindeua & 23 & 13,14 & \multirow{3}{*}{$0,000 *$} \\
\hline Belém & 70 & 40,00 & \\
\hline Benevides & 2 & 1,14 & \\
\hline
\end{tabular}




\begin{tabular}{lrrr}
\hline \hline Gênero & Frequência & Percentual (\%) & P-valor \\
\hline Castanhal & 8 & 4,57 & \\
Interior & 37 & 21,14 & \\
Marituba & 6 & 3,43 \\
Não Informado & 27 & 15,43 \\
Santa Barbara & 1 & 0,57 \\
Santa Izabel & 1 & 0,57 & \\
\hline Tipo de Tratamento Clínico & $\mathbf{1 7 5}$ & $\mathbf{1 0 0 , 0 0}$ & \\
\hline Cirúrgico & 61 & 34,86 & \\
Conservador & 114 & 65,14 & $0,000^{*}$ \\
\hline Total & $\mathbf{4 3 0}$ & $\mathbf{1 0 0 , 0 0}$ & \\
\hline \hline
\end{tabular}

Fonte: Dados resultantes da pesquisa (2015).

* A diferença entre as frequências é significativa ao nível de significância de 0.05.

(1) Teste Qui-quadrado.

A tabela 2 mostra o tipo de trauma apresentado pelos participantes da pesquisa. Verifica-se que os tipos de trauma mais observados foram o traumatismo cranioencefálico (TCE) com 65,71\% (115), o trauma ortopédico representando $17,71 \%$ (31) e as emergências clínicas (desconforto respiratório, acidente vascular encefálico, parada cardiorrespiratória) com 14,86\% (26). Todas as frequências apresentaram diferença significativa, quando comparados entre presença e ausência, ao nível de 0.05 de significância.

Tabela 2-Frequência e percentual dos participantes da pesquisa por Tipo de Trauma. Belém/PA.

\begin{tabular}{lrrrrr}
\hline \multirow{2}{*}{ Tipo de Trauma } & \multicolumn{2}{c}{ Ausência } & \multicolumn{2}{c}{ Presença } & \multirow{2}{*}{ P-valor } \\
\cline { 2 - 5 } & $\mathrm{N}$ & \multicolumn{1}{c}{$\%$} & $\mathrm{~N}$ & \multicolumn{1}{c}{$\%$} & \multicolumn{2}{c}{-v } \\
\cline { 2 - 5 } Traumatismo Cranioencefálico & 60 & 34,29 & 115 & 65,71 & $<0.0001^{*}$ \\
Traumatismo Raquimedular & 169 & 96,57 & 6 & 3,43 & $<0.0001^{*}$ \\
Toraco Abdominal & 155 & 88,57 & 20 & 11,43 & $<0.0001^{*}$ \\
Ortopédico & 144 & 82,29 & 31 & 17,71 & $<0.0001^{*}$ \\
Face e Pescoço & 153 & 87,43 & 22 & 12,57 & $<0.0001^{*}$ \\
Politrauma & 153 & 87,43 & 22 & 12,57 & $<0.0001^{*}$ \\
Emergências clínicas & 149 & 85,14 & 26 & 14,86 & $<0.0001^{*}$ \\
Outros & 174 & 99,43 & 1 & 0,57 & $<0.0001^{*}$ \\
\hline \hline
\end{tabular}

Fonte: Dados resultantes da pesquisa (2015).

* A diferença entre as frequências é significativa ao nível de significância de 0.05.

${ }^{1}$ TesteQui-quadrado de Pearson.

A tabela 3 mostra a região corporal do trauma apresentado pelos participantes da pesquisa. Observa-se que a maioria, $74,86 \%$ (131) apresenta trauma na região da cabeça, seguido da região do tórax com $14,86 \%$ (26) e
$14,29 \%$ (25) com trauma nos membros inferiores (MMII). Todas as frequências apresentaram diferença significativa, quando comparados entre presença e ausência, ao nível de 0.05 de significância. 
Tabela 3-Frequência e percentual dos participantes da pesquisa por região corporal do trauma. Belém/PA

\begin{tabular}{|c|c|c|c|c|c|}
\hline \multirow{2}{*}{ Região Corporal do Trauma } & \multicolumn{2}{|c|}{ Ausência } & \multicolumn{2}{|c|}{ Presença } & \multirow{2}{*}{ P-valor } \\
\hline & $\mathrm{N}$ & $\%$ & $\mathrm{n}$ & $\%$ & \\
\hline Cabeça & 44 & 25,14 & 131 & 74,86 & $<0.0001^{*}$ \\
\hline Tórax & 149 & 85,14 & 26 & 14,86 & $<0.0001^{*}$ \\
\hline Abdômen & 168 & 96,00 & 7 & 4,00 & $<0.0001 *$ \\
\hline Membros Superiores & 162 & 92,57 & 13 & 7,43 & $<0.0001 *$ \\
\hline Membros Inferiores & 150 & 85,71 & 25 & 14,29 & $<0.0001 *$ \\
\hline Pelve & 174 & 99,43 & 1 & 0,57 & $<0.0001 *$ \\
\hline Politrauma & 152 & 86,86 & 23 & 13,14 & $<0.0001 *$ \\
\hline Pescoço & 168 & 96,00 & 7 & 4,00 & $<0.0001 *$ \\
\hline
\end{tabular}

Fonte: Dados resultantes da pesquisa (2015).

* A diferença entre as frequências é significativa ao nível de significância de 0.05 .

${ }^{1}$ TesteQui-quadrado de Pearson.

Quanto a classificação de risco dos participantes da pesquisa verifica-se que $62,3 \%$ (109) foram classificados com risco vermelho, 35,4\% (62) receberam classificação de risco de cor amarelo e 2,3\% (4) de cor verde. As frequências apresentaram diferença significativa, quando comparados entre ostipos de risco (por cor), ao nível de 0.05 de significância.
A tabela 4 mostra o tipo de mecanismo que causou a lesão dos participantes da pesquisa. Os mecanismos mais frequentes foram: acidente de moto 32,57\% (57), ferimento por arma de fogo $16 \%$ (28), clínico $13,14 \%$ (23) e atropelamento $10,86 \%$ (19). As frequências apresentaram diferença significativa, quando comparados os tipos de mecanismo que causaram lesão, ao nível de 0.05 de significância.

Tabela 4- Frequência e percentual dos participantes da pesquisa por Mecanismo de Lesão. Belém/PA.

\begin{tabular}{|c|c|c|c|c|c|}
\hline \multicolumn{5}{|c|}{ Mecanismo de Lesão } & \multirow{2}{*}{$\begin{array}{c}\text { P- } \\
\text { valor }^{(1)}\end{array}$} \\
\hline & & Frequência & Percentual (\%) & $\begin{array}{c}\text { Percentual } \\
\text { Acumulado }(\%)\end{array}$ & \\
\hline \multirow{13}{*}{$\begin{array}{l}\text { Mecanismo } \\
\text { de Lesão }\end{array}$} & Acidente carro & 3 & 1,71 & 1,71 & \multirow{13}{*}{$0,000 *$} \\
\hline & Acidente trabalho/doméstico & 1 &, 57 & 2,29 & \\
\hline & Agressão Física & 8 & 4,57 & 6,86 & \\
\hline & Atropelamento & 19 & 10,86 & 17,71 & \\
\hline & Clínico & 23 & 13,14 & 30,86 & \\
\hline & Ferimento por arma branca & 8 & 4,57 & 35,43 & \\
\hline & Ferimento por arma de fogo & 28 & 16,00 & 51,43 & \\
\hline & Acidente de moto & 57 & 32,57 & 84,00 & \\
\hline & Outros & 1 &, 57 & 84,57 & \\
\hline & Queda da própria altura & 9 & 5,14 & 89,71 & \\
\hline & Queda de altura & 16 & 9,14 & 98,86 & \\
\hline & Queimadura & 2 & 1,14 & 100,00 & \\
\hline & Total & 175 & 100,00 & & \\
\hline
\end{tabular}

Fonte: Dados resultantes da pesquisa (2015).

* A diferença entre as frequências é significativa ao nível de significância de 0.05 .

(1) Teste Qui-quadrado. 
A tabela 5 mostra os procedimentos realizados no atendimento fisioterapêutico. Verifica-se que os procedimentos mais realizados por fisioterapeutas no setor de pronto atendimento foram: o controle de ventilação mecânica $88 \%$ (154), aspiração endotraqueal $60,57 \%$ (106), montagem de ventilação mecânica 49,14\% (86), transporte intra-hospitalar 42,29\% (74), técnicas de fisioterapia respiratória $31,43 \%$

(55).

Tabela 5-Frequência e percentual dos participantes da pesquisa por Tipo de Atendimento Fisioterapêutico. Belém/PA.

\begin{tabular}{lrrrrr}
\hline \multirow{2}{*}{ Procedimentos Fisioterapêuticos } & \multicolumn{2}{c}{ Ausência } & \multicolumn{2}{c}{ Presença } & \multirow{2}{*}{ P-valor } \\
\cline { 2 - 5 } & $\mathrm{N}$ & $\%$ & $\mathrm{n}$ & \multicolumn{1}{c}{ \% } & \\
\hline Montagem de ventilador mecânico & 89 & 50,86 & 86 & 49,14 & $<0.0001^{*}$ \\
Controle de ventilação mecânica & 21 & 12,00 & 154 & 88,00 & $<0.0001^{*}$ \\
Técnicas de Fisioterapia Respiratória & 120 & 68,57 & 55 & $31,43<0.0001^{*}$ \\
Ventilação Mecânica Não-invasiva & 171 & 97,71 & 4 & $2,29<0.0001^{*}$ \\
Ressuscitação Cardiopulmonar & 154 & 88,00 & 21 & $12,00<0.0001^{*}$ \\
Participação na Intubação & 123 & 70,29 & 52 & $29,71<0.0001^{*}$ \\
Oxigenioterapia & 169 & 96,57 & 6 & $3,43<0.0001^{*}$ \\
Aspiração endotraqueal & 69 & 39,43 & 106 & $60,57<0.0001^{*}$ \\
Transporte intrahospitalar & 101 & 57,71 & 74 & $42,29<0.0001^{*}$ \\
Desmame & 172 & 98,29 & 3 & $1,71<0.0001^{*}$ \\
Hiperinsuflação manual & 142 & 81,14 & 33 & $18,86<0.0001^{*}$ \\
\hline \hline
\end{tabular}

Fonte: Dados resultantes da pesquisa (2015).

* A diferença entre as frequências é significativa ao nível de significância de 0.05 .

${ }^{1}$ TesteQui-quadrado de Pearson.

Dentre os procedimentos menos realizados estão: desmame da ventilação mecânica 1,71\% (3), ventilação não invasiva $2,29 \%$ (4) e oxigenioterapia $3,43 \%$ (6).

A internação na unidade de terapia intensiva (UTI) foi o destino da maioria dos pacientes $68,5 \%$ (120), seguido do óbito em 24horas 18,29\% (32), óbito após 24 horas $9,71 \%$ (17) e com menos frequência a internação na enfermaria $2,86 \%$ (5) e transferência pra outro hospital/serviço $0,57 \%$ (1). Esta distribuição de frequências é significativa ao nível de 0.05 .

\section{Discussão}

Desde a implantação do serviço de urgência e emergência no setor hospitalar o perfil de atendimento vem se modificando, sobretudo pela inclusão de uma equipe multidisciplinar composta de médicos, enfermeiros, psicólogos, nutricionistas, assistentes sociais, fonoaudiólogos e fisioterapeutas (BRASIL, 2009; TAQUARY, ATAIIDE, VITORINO, 2013; GARLET et al, 2009).

A fisioterapia na urgência e emergência visa dar suporte a pacientes com complicações cardiorrespiratórias e motoras, contribuindo com a maior eficácia do atendimento prestado aos pacientes que adentram este setor (BRASIL, 2009).

Os pacientes atendidos por fisioterapeutas no pronto atendimento do HMUE correspondem em sua maioria ao gênero masculino na faixa etária de 19 a 29 anos, seguida da faixa etária de 30 a 39 anos, procedentes da cidade de Belém do Pará, 
tendo como terapêutica escolhida o tratamento clínico conservador.

Este perfil assemelha-se ao encontrado na pesquisa de Simons(2008), na qual o maior percentual dos atendimentos realizados em um serviço de urgência e emergência foi constituído de usuários do sexo masculino $(54,5 \%)$ com maior percentual na faixa etária entre 19 e 29 anos (21,6\%), seguido pelo grupo entre 30 e 39 anos (16,5\%).

Semelhantemente, o estudo de Neves et al (2011) identificou o perfil das vítimas de violências e acidentes atendidas em serviços de emergência do Sistema Único de Saúde (SUS) em 2011 onde os indivíduos do sexo masculino representaram a maior proporção entre os atendimentos: $64,2 \%$ dos atendidos por acidentes e 70,4\% dos atendidos por envolvimento em atos de violência. Entre os atendimentos tanto por acidentes quanto por violência, a faixa etária mais frequente foi a de 20 a 39 anos com $39,3 \%$ e $56,9 \%$ respectivamente.

Estes dados podem ser relacionados, entre outras causas, pela maior ocorrência de traumas e acidentes (causas externas) no sexo masculino. A predominância de atendimentos nas faixas etárias mais jovens, especialmente de 19 a 39 anos, está correlacionada com o maior percentual de mortalidade por acidentes, homicídios e agressões em indivíduos jovens, sobretudo do sexo masculino (SIMONS, 2008).
O traumatismo cranioencefálico (TCE), se destacou como o tipo de trauma mais frequente. As regiões corporais mais afetadas foram: cabeça, seguida da região do tórax e membros inferiores. Os mecanismos de lesão mais observados foram acidente de moto e ferimento por arma de fogo (FAF).

Em uma pesquisa com pacientes atendidos em um hospital referência em trauma em Curitiba, Credo e Felix (2012)identificaram que os principais mecanismos de trauma foram os acidentes de trânsito, as quedas e a violência, representando 50,3\% (403), 22,5\% (181), e 21,2\% (170) respectivamente. Nos acidentes de trânsito, as colisões e os atropelamentos foram as principais causas de trauma, sendo que os motociclistas representaram aproximadamente 60\% (240) das vítimas. As fraturas foram identificadas como as lesões mais frequentes, seguidas pelo traumatismo cranioencefálico.

O TCE é um problema de saúde pública de grande magnitude e tem provocado fortes impactos na morbimortalidade da população. Os acidentes e a crescente violência urbana são fatores explicativos importantes na ocorrência do problema (MOLL, 2015; LIMA et al, 2012)

Um estudo realizado por Lopes, Costa e Carvalho (2013) identificou que os acidentes de trânsito foram os principais causadores do TCE em pacientes atendidos no Hospital de Urgências de Teresina. 
Destacando-se em particular os acidentes de moto, que se evidenciam em termo de quantidade, independente da gravidade do TCE ocupando sempre o primeiro lugar com os seguintes percentuais: 63,19\% (TCE leve), $68,43 \%$ (TCE moderado) e 65,00\% (TCE grave).

O perfil dos pacientes e o motivo pelo qual deram entrada no serviço de pronto atendimento encontrados nesta pesquisa divergem de outros estudos que analisaram o perfil de pacientes atendidos por fisioterapeutas em serviços de urgência e emergência.

Um estudo realizado por Gonçalves (2014) no setor de urgência e emergência de um hospital público em Minas geraisidentificou que dos105 pacientes analisados 51,43\% ( $\mathrm{n}=54)$ eram do gênero feminino. A idade média foi de68 anos e as hipóteses diagnósticas de maior incidência foram sepse de foco pulmonar em 10,48\% $(n=11)$ e acidente vascular encefálico (AVE) isquêmico ou hemorrágico em $10,48 \%$ $(n=11)$.

Em outro estudo, Ogawaet al (2009) avaliaram 192 pacientesque deram entrada no serviço de emergência do Hospital São Paulo/UNIFESP, com idade média de 65 anos, sendo $51 \%$ do sexo feminino. Os diagnósticos de entrada mais frequentes foram:precordialgia em 35 (18\%) casos, pneumonia em 29 (15\%) casos e insuficiência cardíaca congestiva descompensada em 20 $(10 \%)$ casos.
Atribui-se esta diferença ao fato do HMUE ser uma referência em trauma, portanto recebe em maior parte, pacientes jovens, do sexo masculino onde a incidência de acidentes e violência é maior, como já citado anteriormente.

Um perfil de pacientes pediátricos atendidos por fisioterapeutas foi identificado por Taquary, Ataíde e Vitorino (2013) em estudo realizado no Serviço de Referência em Urgência Pediátrica (SERUPE) do Hospital das Clínicas da Universidade Federal de Goiás (HC/UFG), no qual foram avaliados 47 pacientes, 53,2\% $(n=25)$ do sexo masculino, idade média de 5,2 $\pm 4,1$ anos. As hipóteses diagnósticas mais identificadas foram doenças respiratórias em 69,4\% ( $\mathrm{n}=43), 32,60 \%$ em crise asmática, seguido de $30,20 \%$ de pneumonia.

Esses estudos evidenciam a atuação do fisioterapeuta em pacientes com diferentes perfis, perpassando desde emergências cardiovasculares, respiratórias até a urgência e emergência no trauma, abrangendo pacientes de todas as faixas etárias.

No presente estudo a classificação de risco vermelho foi a mais presente com $62,3 \%$ (109), seguido da classificação de risco amarelo $35,4 \%$ (62) e apenas 2,3\% (4) receberam classificação de risco verde. O estudo de Picolliet al (2013) objetivou avaliar as indicações para a inserção do fisioterapeuta na emergência de um hospital público na cidade de Porto Alegre e identificou-se que de 
um total de 570 pacientes $189(33,1 \%)$ necessitaram de internação na sala vermelha (urgência absoluta) e 381(66,8\%) sala laranja (muito urgente).

Estes dados sugerem a complexidade dos pacientes atendidos por fisioterapeutas em serviços de urgência e emergência. Uma tentativa de modificar os desfechos negativos seria a inserção de um profissional fisioterapeuta, para atuar, principalmente, em situações agudas que aumentam a morbimortalidade dos pacientes (PICCOLI et al, 2013).

No que diz respeito à atuação do fisioterapeuta no pronto atendimento do HMUE, verificou-se que os procedimentos mais realizados foram: controle de ventilação mecânica, aspiração endotraqueal, montagem de ventilação mecânica, transporte intrahospitalar e técnicas de fisioterapia respiratória.

Outros estudos identificaram que a oxigenioterapia, o controle da ventilação mecânica e técnicas de fisioterapia respiratória foram os procedimentos fisioterapêuticos mais aplicados no setor de urgência e emergência (PICCOLI et al, 2013; OGAWA et al, 2009; TAQUARY, ATAÍDE, VITORINO, 2013, GONÇALVES, 2014).

A oxigenoterapia consiste na administração de oxigênio acima da concentração ambiental normal.A indicação mais comum para sua utilização é a insuficiência respiratória aguda (IRpA) (KOCK et al, 2014). Nesta pesquisa, o fisioterapeuta aplicou a oxigenoterapia em apenas 3,43\% $(\mathrm{n}=06)$ dos pacientes. O fato desta terapêutica não ser um recurso utilizado apenas por fisioterapeutas pode explicar este resultado, além disto, grande parte da população em estudo fez uso de suporte ventilatório invasivo.

O suporte ventilatório invasivo tem como objetivo restabelecer o equilíbrio entre a demanda e oferta de oxigênio, além de diminuir o trabalho da musculatura respiratória. A fisioterapia tem uma importante atuação em pacientes quenecessitam de suporte ventilatório, pois auxilia na condução da ventilação mecânica, desde o preparo e ajuste do ventilador artificial à prótese (tubo orotraqueal ou cânula de traqueostomia) até a evolução do paciente, interrupção e desmame do suporte ventilatório. (MOREIRA et al, 2015; RODRIGUES et al, 2012, NAUE et al, 2014, PRESSUS et al, 2015).

Apesar dos benefícios daventilação mecânica invasiva, os pacientes também estão sujeitos aos efeitos deletérios da mesma. Tais como o comprometimento do mecanismo de transporte e depuração mucociliar que leva a estase de secreções nas vias aéreas com consequente obstrução que podem causar atelectasias e favorecer a proliferação de microorganismos e infecções respiratórias (NAUE et al, 2014).

Nesse contexto, as técnicas de fisioterapia respiratória são aplicadas 
rotineiramente com objetivo de higienizar as vias aéreas, melhorar trocas gasosas, aumentar o fluxo aéreo e prevenir possíveis patologias respiratórias, podendo ser classificadas em técnicas de higiene brônquica e manobras de reexpansão pulmonar (LIEBANO etal, 2009; JERRE, 2007).

As manobras mais comuns são compressão torácica, hiperinsuflação manual, aspiração traqueal, bloqueio torácico e compressão descompressão. A seleção da técnica depende da situação encontrada, bem como o seu objetivo terapêutico, são condutas bastanteutilizadas nos atendimentosfisioterapêuticos (ALVES, 2012; ROSA etal, 2007, FRANÇA et al, 2012).

Neste estudo o transporte intrahospitalar teve a participação do fisioterapeuta em 42,29\% $(n=74)$ dos casos. Este ocorre principalmente para o centro cirúrgico e para realização de exames complementares, sobretudo a tomografia computadorizada (TC). É um período de instabilidade e riscos, com possibilidade de intercorrências, portanto requer a estabilização prévia do paciente, monitorização, equipamentos adequados e uma equipe bem treinada (MENEGUIN; ALEGRE; LUPP, 2014).

Com o desenvolvimento da fisioterapia respiratória e da intervenção fisioterapêutica no paciente grave, o fisioterapeuta passou a fazer parte legalmente da equipe de transporte. Seus conhecimentos, sobretudo em relação à ventilação mecânica e monitorização respiratória são altamente úteis para a estabilização do paciente, favorecendo a segurança do transporte (VEGA, 2012).

O uso de ventilação mecânica não invasiva (VNI) na urgência e emergência cresceu muito nos últimos anos devido fortes evidências de seus efeitos positivos em disfunções respiratórias (VEGA, 2012; SILVEIRA et al, 2011; SARMENTO, 2010).

Porém nesta pesquisa a VNI foi aplicada apenas em 4 (2,29\%) pacientes, assim como no estudo de Gonçalves (2014) onde também foi aplicada apenas em 4 $(3,80 \%)$ pacientes. Uma hipótese para estes resultadosseria o grande número de pacientes que necessitaram de ventilação mecânica invasiva devido à gravidade e complexidade dos traumas, sobretudo os pacientes que sofreram TCE, pois muitos apresentam importante rebaixamento do nível de consciência, evoluindo com insuficiência respiratória necessitando de intubação traqueal e suporte ventilatório artificial (LIMA et al, 2012).

O destino da maioria dos pacientes foi a internação na Unidade de Terapia Intensiva (UTI) com percentual de 68,5\% (120), corroborando dados dos estudos de Piccoliet al (2013); Camerroet al (2015) e Neves et al (2011).

$\mathrm{O}$ atendimento em unidades de emergência é caracterizado pela 
imprevisibilidade, sobretudo nos grandes centros urbanos, onde a demanda de pacientes é alta. Ressalta-se a importância do trabalho em equipe multiprofissional, na qual $o$ fisioterapeuta é de extrema importância para melhorar a qualidade do atendimento prestado, buscando a preservação da vida (TAQUARY; ATAÍDE; VITORINO, 2013).

\section{Conclusão}

A atuação da Fisioterapia no setor de pronto atendimento visa amenizar os sinais e sintomas clínicos, incluindo os respiratórios realizando procedimentos como controle de ventilação mecânica, aspiração traqueal, montagem de ventilação mecânica, transporte intra-hospitalar, técnicas de fisioterapia respiratória, entre outras condutas a fim de contribuir para a estabilização dos pacientes e otimizar o tratamento clínico.

$O$ perfil de pacientes atendidos no setor de pronto atendimento do HMUE é constituído de pacientes adultos jovens com predominância do sexo masculino. O tipo de trauma de maior incidência foi o TCE e os acidentes de moto como o mecanismo de trauma de maior percentual.

As pesquisas envolvendo a atuação da fisioterapia nos setores de urgência e emergência ainda são escassas. Sugere-se a realização de estudos que avaliem os impactos da intervenção fisioterapêutica em unidades de urgência e emergência.

\section{Referências}

1. ALBINO, RM; GROSSEMAN, S; RIGGENBACH, V. Classificação de risco: Uma necessidade inadiável em um serviço de emergência de qualidade. Arquivos Catarinenses de Medicina. v. 36, n. 4, p. 70-75. 2007. Disponível em: <http://www.acm.org.br/revista/pdf/art igos/523.pdf $>$. Acesso em: 06 mar. 2015.

2. ALVES, NA. A importância da atuação do fisioterapeuta no ambiente hospitalar. Sistema Anhanguera de Revistas Eletrônicas. 2012; 16 (6), p. 173-184. Acesso em: 20 mai.2016. Disponível em: <http://pgsskroton.com.br/seer/index.p hp/ensaioeciencia/article/viewFile/275 0/2607>.

3. BRASIL. Ministério da saúde. Secretaria de Atenção à Saúde. Política Nacional de Humanização da Atenção e Gestão do SUS.

Acolhimento e Classificação de Risco nos Serviços de Urgência. Brasília: Ministério da Saúde, 2009. Acesso em: 06 mar. 2015. Disponível em: <http://bvsms.saude.gov.br/bvs/public acoes/acolhimento_classificaao_risco_ servico_urgencia.pdf $>$.

4. CAMERRO, A. et al.Perfil do atendimento de serviços de urgência e emergência. Revista Fafibe On-Line, Bebedouro SP, 8 (1): 515-524, 2015. Acesso em: 07 mar.2015. Disponível em:

$<$ http://unifafibe.com.br/revistasonline /arquivos/revistafafibeonline/sumario/ 36/10112015195658.pdf>.

5. CREDO, PF; FELIX,JVC. Perfil dos pacientes atendidos em um hospital de referência ao trauma em Curitiba: implicações para a enfermagem. Revista Cogitare Enfermagem. 2012 
Jan/Mar; 17(1):126-31. Acesso em: 19 mai.2016. Disponível em: < http://revistas.ufpr.br/cogitare/article/d ownload/26385/17578>.

6. FRANÇA, EET. et al. Fisioterapia em pacientes críticos adultos: recomendações do Departamento de Fisioterapia da Associação de Medicina Intensiva Brasileira. Revista Brasileira de Terapia Intensiva. 2012; 24(1):6-22. Acesso em: 25 mai.2016. Disponível em: <http://www.scielo.br/pdf/rbti/v24n1/ 03.pdf $>$.

7. FUKUJIMA, MM. et al. Atualização em fisioterapia na emergência.[online]. São Paulo: Unifesp, 2009. 176 p. ISBN 978-8561673-67-3. Acesso em 24 de mar. 2015. Disponível em: <http://books.scielo.org>.

8. GARLET, ER.et al Organização do trabalho de uma equipe de saúde no atendimento ao usuário em situações de urgência e emergência. Revista Texto Contexto Enferm, Florianópolis, v.12, n.2,abr/jun. 2009. Acesso em: 19 mai.2016. Acesso em: $<$ http://www.scielo.br/pdf/tce/v18n2/0 9.pdf $>$.

9. GONÇALVES, ACS. Perfil clínico dos pacientes atendidos pelo serviço de fisioterapia na unidade de urgência e emergência de um hospital público de Minas Gerais. Revista ASSOBRAFIR Ciência. v. 5, n. 3, p. 55-62, dez. 2014. Acesso em: 06 mar. 2015. Disponível em: <http://www.uel.br/revistas/uel/index. php/rebrafis/article/view/18166/15576 $>$.

10. JERRE G. Fisioterapia no paciente sob ventilação mecânica. Revista Brasileira de Terapia Intensiva, v. 19, n. 3, Jul/Set.2007. Acesso em: 24 mai.2016. Disponível em: <http://www.scielo.br/scielo.php?scrip $\mathrm{t}=$ sci_arttext\&pid=S180637132007000800010>.

11. KOCK,KS et al. Adequações dos dispositivos de oxigenoterapia em enfermaria hospitalar avaliadas por oximetria de pulso e gasometria arterial. ASSOBRAFIR Ciência. 2014 Abr;5(1):53-64. Acesso em: 22 mai.2016. Disponível em: <http://www.uel.br/revistas/wrevojs24 6/index.php/rebrafis/article/view/1642 $2>$.

12. LIEBANO, RE. et al. Principais manobras cinesioterapêuticas manuais utilizadas na fisioterapia respiratória: descrição das técnicas. Revista de Ciências Médicas, Campinas, 18(1):35-45, jan./fev., 2009. Acesso em: 21 mai.2016. Disponível em: $<$ http://periodicos.puccampinas.edu.br/seer/index.php/cienci asmedicas/article/view/652>.

13. LIMA, MVC. et al. Perfil Clínico e Desmame Ventilatório de Pacientes Acometidos por Traumatismo CrânioEncefálico. Revista de Neurociências 2012; 20(3):354-359. Acesso em: 22 mai.2016. Disponível em: <http://www.revistaneurociencias.com .br/edicoes/2012/RN2003/original\%20 20\%2003/661\%20original.pdf>.

14. LOPES, RD; COSTA, PP;CARVALHO, FT. Perfil epidemiológico dos pacientes acometidos por trauma cranioencefálico assistidos em um hospital público de Teresina. Revista Brasileira de Neurologia e Psiquiatria. 2013 dez;7(3):80-90. Acesso em: 19 mai.2016. Disponível em: $<$ http://rbnp.emnuvens.com.br/rbnp/art icle/view/27/17>.

15. MENEGUIN, S; ALEGRE, PHC; LUPP,CHB. Caracterização do transporte de pacientes críticos na 
modalidade intra-hospitalar. Acta Paul Enferm. 2014; 27(2):115-9. Acesso em: 25 mai.2016. Disponível em: <http://www.scielo.br/scielo.php?pid= S0103-

21002014000200005\&script=sci_abst ract\&tlng $=\mathrm{es}>$.

16. MOLL, AVS. Perfil do atendimento dos pacientes com traumatismo cranioencefálico nos hospitais de urgência e emergência sob a gestão da Secretaria de Estado de Saúde do Rio de Janeiro [Dissertação]. Rio de Janeiro: Escola Nacional de Saúde Pública Sergio Arouca. ; 2015.

17. MOREIRA, FC.et al. Alterações da mecânica ventilatória durante a fisioterapia respiratória em pacientes ventilados mecanicamente. Revista Brasileira de Terapia Intensiva. 2015 ;27(2):155-160. Acesso em> 19 mai.2016. Disponível em: http://www.scielo.br/scielo.php?pid=S 0103 -

507X2015000200155\&script=sci_abst ract\&tlng $=\mathrm{t}>$.

18. NAUE, WS.et al. Compressão torácica com incremento da pressão em ventilação com pressão de suporte: efeitos na remoção de secreções, hemodinâmica e mecânica pulmonar em pacientes em ventilação mecânica. Jornal Brasileiro de Pneumologia. v.40, n.1, p 55-60. 2014. Acesso em> 23 mai.2016. Acesso em: $<$ http://www.jornaldepneumologia.co m.br/detalhe_artigo.asp?id=2250 $>$.

19. NEVES, ACM.et al. Perfil das vítimas de violências e acidentes atendidas em serviços de urgência e emergência do Sistema Único de Saúde em capitais brasileiras - 2011. Revista Epidemiologia e Serviços de Saúde. Acesso em: 19 mai.2016. Disponível em:

$<$ http://scielo.iec.pa.gov.br/pdf/ess/v2 2n4/v22n4a05.pdf $>$.
20. OGAWA, KYL.et al. Intervenção fisioterapêutica nas emergências cardiorrespiratórias. Revista $\mathrm{O}$ Mundo da Saúde, São Paulo, v. 33, n. 4, p. 457-466, 2009. Acesso em: 06 mar. 2015. Disponível em: $<$ http://www.saocamilosp.br/pdf/mundo_saude/70/457a466.p df $>$.

21. PICCOLI, A. et al.Indicações para Inserção do Profissional Fisioterapeuta em uma Unidade de Emergência. Revista ASSOBRAFIR Ciência. v. 4, n. 1, p. 33-41, 2013. Acesso em: 02 mar. 2015. Disponível em: $<$ http://www.uel.br/revistas/uel/index. php/rebrafis/article/view/13412/12758 $>$.

22. PREUSS, FK.et al Efeitos de dois protocolos de fisioterapia respiratória na mecânica respiratória e parâmetros cardiorrespiratórios de pacientes em ventilação mecânica: estudo piloto. Revista Fisioterapia e pesquisa. 2015;22(3):246-52.

23. RODRIGUES, YCSJ.et al.Ventilação mecânica: evidências para o cuidado de enfermagem. Escola Anna Nery Revista de Enfermagem. 2012 out dez; 16 (4):789-795. Acesso em: 20 mai.2016. Disponível em: $<$ http://www.scielo.br/scielo.php?scrip $\mathrm{t}=$ sci_arttext\&pid=S141481452012000400021>.

24. ROSA, FK.et al. Comportamento da mecânica pulmonar após a aplicação de protocolo de fisioterapia respiratória e aspiração traqueal em pacientes com ventilação mecânica invasiva. Revista Brasileira de Terapia Intensiva, v.19, n 2, Abr/Jun. 2007. Acesso em: 25 mai.2016. Disponível em:

$<$ http://www.scielo.br/scielo.php?scrip $\mathrm{t}=$ sci_arttext\&pid=S0103507X2007000200005>. 
25. SARMENTO GJV. (Org.).

Fisioterapia respiratória no paciente crítico: rotinas clínicas. 3. ed. São

Paulo: Manole, 2010. 675p.

26. SILVEIRA, APC.et al. Comparação do uso da pressão positiva com a fisioterapia convencional e incentivadores respiratórios após cirurgia cardíaca: revisão de literatura. Revista de Medicina de Ribeirão Preto. 2011;44(4): 338-46. Acesso em: 20 mai.2016. Disponível em: $<$ http://revista.fmrp.usp.br/2011/vol44 n4/REV_Compara\%E7\%E3o\%20do\% 20uso\%20da\%20press\%E3o\%20positi va\%20com $\% 20 \mathrm{a} \% 20$ fisioterapia $\% 20 \mathrm{c}$ onvencional.pdf>.

27. SIMONS, DA. Avaliação do perfil da demanda na unidade de emergência em Alagoas a partir da municipalização da saúde e do Programa Saúde da Família [Tese de doutorado em saúde pública]. Recife: Centro de Pesquisas Aggeu Magalhães, Fundação Oswaldo Cruz.; 2008.

28. SIMÕES, CG; URBANETTO, JS; FIGUEIREDO, AEPL. Ação interdisciplinar em serviços de urgência e emergência: uma revisão integrativa. Revista Ciência \& Saúde. São Paulo, v. 6, n. 2, p. 127-134, mai./ago. 2013. Acesso em: 06 mar. 2015. Disponível em <http://revistaseletronicas.pucrs.br/ojs/ index.php/faenfi/article/view/13321/9 714>.

29. TAQUARY, SAS; ATAIIDE, DS; VITORINO, PVO. Perfil clínico e atuação fisioterapêutica em pacientes atendidos na emergência pediátrica de um hospital público de Goiás. Revista Fisioterapia e Pesquisa. v.20, p. 262267, 2013. Acesso em: 03 mar. 2015. Disponível em:
$<$ http://www.scielo.br/pdf/fp/v20n3/11 .pdf $>$

30. VEJA,JM. (Ed.). Tratado de fisioterapia hospitalar: assistência integral ao paciente. São Paulo: Atheneu, 2012. 1221p. 